Pacific Journal of Mathematics

STRUCTURE OF Г-RINGS

S. RAVISANKAR AND U. S. SHULA 


\section{STRUCTURE OF $\Gamma$-RINGS}

\section{T. S. Ravisankar and U. S. Shukla}

In the first part of the present paper, $\Gamma$-rings are studied in the setting of modules. The notion of a module over a $\Gamma$-ring is studied, with the object of developing the notion of a Jacobson-radical for a $\Gamma$-ring via modules. This radical enjoys the usual properties of the corresponding object in rings. A semisimple right Artinian $\Gamma$-ring turns out to be the direct sum of simple ideals; this conclusion is strengthened to include a corresponding decomposition for the $R$-ring $\Gamma$ also in the case of a strongly semisimple strongly right Artinian weak $\Gamma_{N}$-ring. The Jacobson radical of a weak $\Gamma_{N}$-ring $R$ is characterized in different ways, in one of them as the set of all properly quasi-invertible elements of $R$. It is shown how rings, ternary rings and associative triple systems can be considered as weak $\Gamma_{N}$-rings. The present approach provides a uniform module $\mathrm{cum}$ radical theory not only for $\Gamma$-rings, but also for the associative triple systems.

The second part of the paper imbeds any weak $\Gamma_{N}$-ring $R$ into a suitable associative ring $A$. Simplicity and semisimplicity in $R$ and $A$ are shown to be related. The main result of this part which generalizes the classical Wedderburn-Artin theorem for rings to $\Gamma$-rings, characterizes the strongly simple, strongly right Artinian weak $\Gamma_{N}$-rings as the $\Gamma$-rings of rectangular matrices over division rings.

The ring of all square matrices over a division ring plays a vital role in classical ring theory. However, when one considers the set of all rectangular matrices (of the same type), there appears to be no natural way of introducing a binary ring multiplication into it. Various authors like Nobusawa [15], Lister [8] and Hestenes (see [5]) have tried to offset this difficulty by considering a natural ternary multiplication in the set of rectangular matrices; their investigations have led to the respective notions of a $\Gamma_{N}$-ring, associative triple systems of first kind (ternary rings) and of second kind. These three structures provide a suitable setting for the study of rectangular matrices. The above-mentioned authors have obtained some structural results for these structures, results similar to ones for rings. The concept of weak $\Gamma_{N}$-ring introduced in this paper includes all the three above structures, besides rings, as particular cases. Nobusawa considers a notion of semisimplicity for his $\Gamma_{N}$-ring and that does not arise from a radical as in the case of rings. Coppage and Luh [2] have considered a few radicals among which 
the "strongly nilpotent" radical proves adequate as a radical for Nobusawa's semisimplicity. Besides, they also consider a Jacobson radical in this paper; Luh also considers a notion of primitivity in the context of density theorem for $\Gamma$-rings (see [9]). Significantly, both these concepts are introduced without reference to a concept of a module, unlike in ring theory. In the present paper, a suitable notion of a module over a $\Gamma$-ring is introduced and this provides a natural setting for both these concepts. Indeed, the notions of primitivity and Jacobson radical are introduced via modules and the earlier notions are recovered in this context. The present approach also provides a simultaneous radical theory for the the associative triple systems of both the kinds at the same time (an approach of which type has been lacking so far).

1. $\Gamma$-rings and modules over $\Gamma$-rings. Let $\Gamma=\{\alpha, \beta, \gamma, \delta, \cdots\}$ be an additive (abelian) group. A $\Gamma$-ring is an additive group $R=$ $\{x, y, z, \cdots\}$ together with a composition $x \alpha y$ in $R$ defined for $x, y$ in $R$ and $\alpha$ in $\Gamma$ satisfying the following conditions:

(1) $(x+y) \alpha z=x \alpha z+y \alpha z, x(\alpha+\beta) y=x \alpha y+x \beta y, x \alpha(y+z)=$ $x \alpha y+x \alpha z$

(2) $x \alpha(y \beta z)=(x \alpha y) \beta z$.

$R$ will be called a $\Gamma$-ring in the sense of Nobusawa $\left(\Gamma_{N}\right.$-ring) (see [1], [15]), if there is also a similar composition $\alpha x \beta$ in $\Gamma$ defined for $\alpha, \beta$ in $\Gamma$ and $x$ in $R$ satisfying (1),

(1') $(\alpha+\beta) x \delta=\alpha x \delta+\beta x \delta, \alpha(x+y) \beta=\alpha x \beta+\alpha y \beta, \alpha x(\beta+\delta)=$ $\alpha x \beta+\alpha x \delta$

$\left(2^{\prime}\right) \quad(x \alpha y) \beta z=x(\alpha y \beta) z=x \alpha(y \beta z)$

$\left(2^{\prime \prime}\right) \quad(\alpha x \beta) y \delta=\alpha(x \beta y) \delta=\alpha x(\beta y \delta)$ and

(3) $x \alpha y=0$ for all $x, y$ in $R$ implies $\alpha=0$ (Nobusawa condition).

In fact, $\left(1^{\prime}\right)$ and $\left(2^{\prime \prime}\right)$ are redundant in the above definition (see $[10$, p. 72$])$. We shall call a $\Gamma$-ring $R$ to be a weak $\Gamma_{N}$-ring in case the composition $\alpha x \beta$ in $\Gamma$ satisfies $\left(1^{\prime}\right),\left(2^{\prime}\right)$ and $\left(2^{\prime \prime}\right)$ only. Clearly, if $R$ is a weak $\Gamma_{N}$-ring, $\Gamma$ can be thought of as a weak $R_{N}$-ring.

Examples. (1) Let $D$ be a division ring and $M_{p, q}(D)$ denote the additive group of all $p \times q$ matrices whose entries are from $D$.

(a) $M_{p, q}(D)$ is a $\Gamma$-ring with $\Gamma=M_{p, q}(D)$, where the composition is defined by $x \alpha z=x\left(\alpha^{t}\right) z, \alpha^{t}$ denoting the transpose of the matrix $\alpha$, for all $x, y$ and $\alpha$ in $M_{p, q}(D)$.

(b) $M_{p, q}(D)$ is a $\Gamma$-ring with $\Gamma=M_{q, p}(D)$ under the usual matrix multiplication.

(2) Every associative ring $R$ is a $\Gamma$-ring with $\Gamma=R$, but it need not be a $\Gamma_{N}$-ring in general; however, it is easily seen to be a weak $\Gamma_{N}$-ring always. 
(3) Any associative triple system $T$ of first (second) kind with trilinear composition $\left\langle>\right.$ becomes a weak $\Gamma_{N}$-ring $R(=T)$ for $\Gamma=T$ if one defines multiplication in $R$ as $\langle>$ and in $\Gamma$ as $\alpha x \beta=$ $\langle\alpha x \beta\rangle(\alpha x \beta=\langle\beta x \alpha\rangle)$.

An additive subgroup $I$ of a $\Gamma$-ring $R$ is called a right (left) ideal of $R$, if $x \alpha y \in I(y \alpha x \in I)$ for all $x$ in $I, \alpha$ in $\Gamma$ and $y$ in $R$. A right ideal which is a left ideal as well is called a two-sided ideal or simply an ideal. $R$ is said to be simple, if $R \Gamma R \neq 0$ and the only ideals of $R$ are 0 and $R$. As in the classical case of rings, one can easily see that the $\Gamma$-ring in Example $1(b)$ is a simple $\Gamma$-ring.

Let $R$ be a $\Gamma$-ring. An additive (abelian) group $M$ will be called a right $R \Gamma$-module (to be called just $R \Gamma$-module), if there exists a map $\varphi: M \times \Gamma \times R \rightarrow M$ satisfying $(\varphi(m, \alpha, x)$ will be denoted by $m \alpha \cdot x$ in short)

(1) $(m+n) \alpha x=m \alpha x+n \alpha x$,

(2) $m \alpha(x+y)=m \alpha x+m \alpha \cdot y$ and

(3) $m \beta(x \alpha y)=(m \beta x) \alpha y$, for all $x, y$ in $R, \alpha, \beta$ in $\Gamma$ and $m, n$ in $M$.

Examples. (i) Every $\Gamma$-ring $R$ is an $R \Gamma$-module under the right multiplication in the $\Gamma$-ring $R$.

(ii) Let $D$ be a division ring and $D_{p}$ be the set of all $p$-tuples of elements of $D$. Then $D_{p}$ as well as $M_{p, p}(D)$ can be made in a natural way into $R \Gamma$-modules for $\Gamma=M_{p, q}(D)$ and $R=M_{q, p}(D)$.

Let $M$ be an $R \Gamma$-module. For subsets $M_{1}, \Gamma_{1}, R_{1}$ of $M, \Gamma, R$ respectively, we denote by $M_{1} \Gamma_{1} R_{1}$ the additive subgroup of $M$ generated by elements of the form ma:x with $m$ in $M_{1}, \alpha$ in $\Gamma_{1}$ and $x$ in $R_{1}$; same notation also holds for $R$ considered as an $R \Gamma$-module. One can define, as in the classical module case, the notions of submodules, proper submodules, quotient module, homomorphism between $R \Gamma$-modules, kernel of a homomorphism and direct sum of $R \Gamma$-modules etc. Among the usual homomorphism results that hold in the present case, we record the following result for later reference.

Proposition 1.1. Let $N$ be a submodule of $M$. Then there is a one-to-one correspondence between the submodules of $M$ containing $N$ and the submodules of $M / N$.

For an ideal $I$ of a $\Gamma$-ring $R, R / I$, the additive group of cosets of $I$, forms a $\Gamma$-ring in a natural way. If $I$ is a right ideal of $R$ then $I$ is a submodule of the $R \Gamma$-module $R$; as such, $R / I$ can also be considered as an $R \Gamma$-module. We shall call an $R \Gamma$-module $M$ to be irreducible if $M \Gamma R \neq 0$ and if it has no nonzero proper submodules.

The following two results can be easily obtained as in the classical case (see [4]) with slight modifications. 
Lemma 1.2. $M$ is irreducible $\Leftrightarrow$ for $m \neq 0$ in $M, m \Gamma R=M \Leftrightarrow$ for each $m \neq 0$ in $M$ there is an $\alpha$ in $\Gamma$ such that $m \alpha R=M$.

Lemma 1.3 (cf. [4, Lemma 1.1.3]). Let $M$ be an irreducible $R \Gamma$-module. Then $M \cong R / I$ as an $R \Gamma$-module for some maximal right ideal $I$ of $R$. Moreover there exists an $\alpha$ in $\Gamma$ and an $e$ in $R$, such that $x-e \alpha x \in I$ for all $x$ in $R$. On the other hand, for every such maximal right ideal $I$ of $R, R / I$ is an irreducible $R \Gamma$ module.

As in the classical case (see [4]), we shall call a right ideal $I$ of $R$ regular, if there exists an element $e$ in $R$ and an $\alpha$ in $\Gamma$, such that $x-e \alpha x \in I$ for all $x$ in $R$.

An $R \Gamma$-module $M$ is said to be a faithful module, if $M \Gamma x=0$ implies $x=0$ (for $x$ in $R$ ) or equivalently, if the annihilator ideal $A(M)=\{x \in R \mid M \Gamma x=0\}$ is zero. $R$ is said to be primitive, if it admits a faithful irreducible $R \Gamma$-module. An ideal $I$ of $R$ is a primitive ideal if $R / I$ is a primitive $\Gamma$-ring. Then we have

Proposition 1.4. If $M$ is an irreducible $R \Gamma$-module, then $R / A(M)$ is a primitive $\Gamma$-ring.

REMARK 1.5. We note here that there is already a notion of primitivity for $\Gamma$-rings in the literature due to Luh (see [10]) which coincides with ours (as shown below). However, the above definition of a primitive $\Gamma$-ring seems to be a natural one in the context of its usefulness as we shall see presently.

TheORem 1.6. A $\Gamma$-ring $R$ is primitive if and only if $R$ is primitive in the sense of Luh (loc. cit.).

Proof. Let $R$ be a primitive $\Gamma$-ring as defined by Luh. Then $R \Gamma x=0$ will imply $x=0$ and the right operator ring $O_{R}$ of $R$ (see [10]) is primitive. Let $M$ be a faithful irreducible $O_{R}$-module. $M$ becomes an $R \Gamma$-module, if we define $m \alpha x=m[\alpha, x]$, for $\alpha$ in $\Gamma$ and $x$ in $R\left([\alpha, x] \in O_{R}\right.$; see [10]). Clearly $M$ is irreducible as an $R \Gamma$ module. Also, if $M \Gamma x=0$, then $M[\alpha, x]=0$ for each $\alpha$ in $\Gamma$; therefore $[\alpha, x]=0$ for all $\alpha$ in $\Gamma$; this implies that $R[\alpha, x]=0$ for all $\alpha$ in $\Gamma$; hence $R \Gamma x=0$ and consequently $x=0$. Therefore $M$ is a faithful irreducible $R \Gamma$-module; so $R$ is primitive.

Conversely, let $R$ be primitive and $M$ be a faithful irreducible $R \Gamma$-module. $M$ becomes an irreducible $O_{R}$-module if we define $m \Sigma\left[\alpha_{i}, x_{i}\right]=\Sigma m \alpha_{i} x_{i}$. Let now $\Sigma\left[\alpha_{i}, x_{i}\right]$ be in $O_{R}$ such that $M \Sigma\left[\alpha_{i}, x_{i}\right]=$ 0 , i.e., $\Sigma m \alpha_{i} x_{i}=0$ for all $m$ in $M$; in particular $\Sigma(m \beta x) \alpha_{i} x_{i}=0$, for all 
$x$ in $R$ and $\beta$ in $\Gamma ; m \beta \Sigma\left(x \alpha_{i} x_{i}\right)=0$ for all $m$ in $M$ and $\beta$ in $\Gamma$. Now, the fidelity of $M$ implies that $\Sigma\left(x \alpha_{i} x_{i}\right)=0 ; x$ being arbitrary in $R$ it follows from the definition of $O_{R}$ that $\Sigma\left[\alpha_{i}, x_{i}\right]=0$.

2. Jacobson radical. As in the case of a ring, we define the Jacobson radical $J(R)$ of a $\Gamma$-ring $R$ by $J(R)=\cap A(M)$, where $M$ runs over all irreducible $R \Gamma$-modules, if any. In case $R$ does not possess any irreducible $R \Gamma$-module, $J(R)$ is defined to be $R$ itself. Since any irreducible $R \Gamma$-module $M$ is of the form $R / I$ for a maximal regular right ideal $I$ of $R$ (see Lemma 1.3) and then $A(M)=$ $\{x \in R \mid R \Gamma x \subseteq I\}=(R: I)$, we have

Proposition 2.1. $J(R)=\cap(R: I)$, where $I$ runs over all the maximal regular right ideals of $R$.

REMARK 2.2. One can easily see (cf. $[4$, p. 10]) that $(R: I)$ will be the largest (two-sided) ideal of $R$ contained in $I$, in case $I$ is regular. Further, any proper regular right ideal is contained in a maximal regular right ideal (Zorn's lemma).

Proposition 2.3. $J(R)=K \equiv \cap I$, where I runs over all maximal regular right ideals of $R$.

Proof. Since $I$ is regular, $(R: I) \subseteq I$ (by the above Remark); by Proposition 2.1, $J(R) \subseteq K$. For the reverse inclusion we first note that for any element $x$ in $K$ and any $\beta$ in $\Gamma$, the right ideal $S=$ $\{x \beta y+y \mid y$ in $R\}$ coincides with $R$ (this can again be proved as in [4, p. 11]). Let now, if possible, $J(R) \subsetneq K$. Then there exists an irreducible $R \Gamma$-module $M$ such that $M \Gamma K \neq 0$. In particular, $m \alpha K \neq 0$ for some $m(\neq 0)$ in $M$ and some $\alpha$ in $\Gamma$. By irreducibility of $M$, one has that $m \alpha K=M$. Let $t$ be in $K$ such that $m \alpha t=-m$. As mentioned above $\{t \alpha y+y \mid y \in R\}=R$. Since $-t$ is in $R$, there exists an $z$ in $R$ such that $t+z+t \alpha z=0$. Then we have

$$
\begin{aligned}
0 & =m \alpha(t+z+t \alpha z)=m \alpha t+m \alpha z+(m \alpha t) \alpha z \\
& =-m+m \alpha z-m \alpha z=-m,
\end{aligned}
$$

a contradiction.

We shall call an element $x$ in $R$ to be $\alpha$-right quasi-regular $(\alpha$-rt.q.r.) with $\alpha$-right quasi-inverse $y$, in case $x+y+x \alpha y=0$. We note indeed that the elements of $J(R)$ are $\alpha$-rt.q.r. for every $\alpha$ in $\Gamma$, as is clear from the above proof. $x$ will be said to be right quasi-regular (rt.q.r.), if it is $\alpha$-right quasi-regular for each $\alpha$ in $\Gamma$. 
Similar notions of $\alpha$-left quasi-regularity etc. can also be clearly introduced. An element $x$ in $R$ is then called $\alpha$-quasi-regular (quasiregular), if it is both $\alpha$-left as well $\alpha$-rt.q.r. (for each $\alpha$ in $\Gamma$ ). An ideal (left, right or two-sided) is $\alpha$-rt.q.r. (rt.q.r., quasi-regular) if each of its elements is so.

REMARK 2.4. (i) $J(R)$ is a right quasi-regular ideal of $R$.

(ii) $x$ in $R$ is $\beta$-rt.q.r. if and only if $\{x \beta y+y \mid y \in R\}=R$.

(iii) The $\alpha$-left and $\alpha$-right quasi-inverses of an $\alpha$-quasi-regular element are one and the same (cf. [12, p. 111]).

(iv) If for an $x$ in $R,-x \alpha x$ is $\alpha$-right $q . r$. with quasi-inverse $y$, then $x$ is also $\alpha$-rt.q.r. with rt. quasi-inverse $-x+y-x \alpha y$ (cf. [6, Lemma 19.1]).

( $v)$ Sum of two $\alpha-r t . q . r$. right ideals of $R$ is again $\alpha-r t . q . r . ;$ indeed any finite sum (in fact, any algebraic sum) of $\alpha-r t . q . r .(r t . q . r$. right ideals is again $\alpha$-rt.q.r.(rt.q.r.).

If we denote by $J_{\alpha}(R)\left(J_{*}(R)\right)$ the sum of all $\alpha-r t . q . r$. (right quasiregular) right ideals of $R$, then $J_{\alpha}(R)\left(J^{*}(R)\right)$ will itself be a $\alpha$ $r t . q . r .(r t . q . r$.$) right ideal in view of the above remark. We also$ have $J_{*}(R)=\bigcap_{\alpha \in \Gamma} J_{\alpha}(R)$. Further, any $\alpha$-rt.q.r. right ideal is also $\alpha$-left quasiregular (easily seen as in the case of rings). Hence $J_{\alpha}(R)\left(J_{*}(R)\right)$ is also a $\alpha$-quasi-regular right ideal. Using the last remark one finds that $J(R)$ is also a quasi-regular ideal. Besides, $J(R)$ contains all quasi-regular right ideals of $R$ (this easily follows as in [4, Theorem 1.2.3]). Thus we have

THEOREM 2.5. $J(R)$ is the unique maximal quasi-regular ideal of $R$ which contains all quasi-regular right ideals (hence also twosided ideals) of $R$.

Since $J(R) \subseteq J_{\alpha}(R)$ for each $\alpha$ in $\Gamma$ and $J_{*}(R)$ is a quasi-regular right ideal of $R$, we have the

Corollary 2.6. For a $\Gamma$-ring $R, J(R)=J_{*}(R)=\bigcap_{\alpha \in \Gamma} J_{\alpha}(R)$.

REMARK 2.7. It is clear that one could have started with left $R \Gamma$-modules and defined a (left) Jacobson radical and obtained left analogues of the earlier results. However, Theorem 2.5 shows that we would have still obtained the same Jacobson radical.

Proposition 2.8 (cf. [4, Theorem 1.2.5]). For an ideal $I$ of $R, J(I)=J(R) \cap I$.

We also have the following elementwise characterization of the Jacobson radical. 
Proposition 2.9. $J(R)=X \equiv\{x \in R \mid x \Gamma R$ is a quasi-regular right ideal in $R\}=\{z \in R \mid x \alpha z \beta y$ is quasi-regular for all $x, y$ in $R$ and $\alpha, \beta$ in $\Gamma\}$.

Proof. Clearly $J(R) \leqq X$. Now, $X$ is easily seen to be a right ideal of $R$. Further, for any $x$ in $X,-x \alpha x$ (belonging to $x \alpha R$ ) is $\alpha$-rt.quasi-regular for all $\alpha$ in $\Gamma ; x$ is $\alpha-r t$.quasi-regular for all $\alpha$ in $\Gamma$ (by Remark 2.4 (iv)). Thus $X$ is a quasi-regular right ideal; $X \subseteq J(R)$. The other part is similar.

We note that there is already a notion of Jacobson radical for a $\Gamma$-ring in the literature due to Luh and Coppage [2]. Indeed they call an element $y$ in $R$ to be right quasi-regular, if for each $\beta$ in $\Gamma$ there exist $\alpha_{i}$ in $\Gamma$ and $x_{i}$ in $R(i=1,2, \cdots, n)$ such that $x \beta y+$ $\Sigma x \alpha_{i} x_{i}-\Sigma x \beta y \alpha_{i} x_{i}=0$ for all $x$ in $R$. Then Jacobson radical is there defined to be (the ideal) $\mathscr{J}(R)=\{x \in R \mid$ the two-sided ideal $\langle x\rangle$ generated by $x$ consists of right quasi-regular elements\}. If $y$ is right quasi-regular in our sense, then for each $\beta$ in $\Gamma$ there exists an $z$ in $R$ such that $y+z-y \beta z=0$; hence $x \beta y+x \beta z-x \beta y \beta z=0$ for all $x$ in $R$, i.e., $y$ is right quasi-regular in the above sense of [2]. However we are unable to conclude anything about the reverse implication. All the same we have

\section{THEOREM 2.10. For a $\Gamma$-ring $R, J(R)=\mathscr{J}(R)$.}

Proof. It is clear that $J(R) \subseteq \mathscr{J}(R)$. Let, if possible, $J(R) \varsubsetneqq$ $\mathscr{J}(R)$; hence there exists an irreducible $R \Gamma$-module $M$ such that $M \Gamma \mathscr{J}(R) \neq 0$; hence $m \alpha \mathscr{J}(R) \neq 0$ for some $m \neq 0$ in $M$ and $\alpha$ in $\Gamma$; by irreducibility of $M, m \alpha \mathcal{J}(R)=M$. Let $m=m \alpha x$ for an $x$ in $\mathscr{J}(R)$. Since $x$ is in $\mathscr{J}(R)$ there exist $\mu_{i}$ in $\Gamma$ and $x_{i}$ in $R(i=$ $1,2, \cdots, n)$ such that $x \alpha x+\sum_{i=1}^{n} x \mu_{i} x_{i}-\sum_{i=1}^{n} x \alpha x \mu_{i} x_{i}=0$; therefore

$$
\begin{aligned}
0 & =m x\left(x \alpha x+\sum_{i=1}^{n} x \mu_{i} x_{i}-\sum_{i=1}^{n} x \alpha x \mu_{i} x_{i}\right) \\
& =(m \alpha x) \alpha x+\sum_{i=1}^{n}(m \alpha x) \mu_{i} x_{i}-\sum_{i=1}^{n}((m x x) \alpha x) \mu_{i} x_{\imath} \\
& =m+\sum_{i=1}^{n} m \mu_{i} x_{\imath}-\sum_{i=1}^{n} m \mu_{i} x_{i}=m,
\end{aligned}
$$

a contradiction.

An element $x$ in a $\Gamma$-ring $R$ is said to be nilpotent if for each $\alpha$ in $\Gamma$, there exists a positive integer $n$ such that $(x \alpha)^{n} x=0 . \quad$ An ideal $I$ (left, right or two-sided) is said to be nil if each of its elements is nilpotent. The ideal $I$ is said to be nilpotent if $(I \Gamma)^{k} I=0$ for some $k$. It is easily seen that a nil ideal is quasi-regular and 
hence is contained in $J(R)$. For right Artinian $\Gamma$-rings (defined in the usual way) one also has

Proposition 2.11 (cf. [4, Theorem 1.3.1]). If $R$ is right Artinian, then $J(R)$ is nilpotent. In fact, $J(R)$ coincides with the nilradical (sum of all nil ideals of $R$ (see [2])).

As in the case of rings we shall call a $\Gamma$-ring $R$ to be semisimple if its Jacobson radical is zero. A proof as in the case of rings yields the following expected result.

Proposition 2.12. For a $\Gamma$-ring $R, R / J(R)$ is semisimple.

It is clear that one can introduce a notion of subdirect sum of $\Gamma$-rings ( $\Gamma$ being same for all of them) in an exactly analogous manner as for rings (see [4]). The following result is then deduced on exactly same pattern (loc. cit.).

Proposition 2.13. A nonzero $\Gamma$-ring is semisimple if and only if it is a subdirect sum of primitive $\Gamma$-rings.

We recall that Nobusawa (see [15]) has called a $\Gamma$-ring $R$ to be semisimple (we shall call it $N$-semisimple), if for each $x(\neq 0)$ in $R$, there exists an $\alpha$ in $\Gamma$ such that $x \alpha x \neq 0$. We note here that the Jacobson radical $J(R)$ plays the role of radical for $N$-semisimplicity also, for an important class of $\Gamma$-rings $R$. Indeed we have

Theorem 2.14. Let $R$ be a right Artinian weak $\Gamma_{N}$-ring. Then $R$ is semisimple if and only if $R$ is $N$-semisimple.

Proof. Let $R$ be semisimple so that $J(R)=0$. If $x(\neq 0)$ is in $R$, then $x \notin J(R)$; there exists an irreducible $R \Gamma$-module $M$ such that $M \Gamma x \neq 0 ; m \alpha x \neq 0$ for some $m$ in $M$ and $\alpha$ in $\Gamma$. By Lemma 1.2, $M=(m \alpha x) \beta R$ for some $\beta$ in $\Gamma$. Hence $m=(m \alpha x) \beta y$ for some $y$ in $R$. Then $0 \neq m \alpha x=((m \alpha x) \beta y) \alpha x=m \alpha(x(\beta y \alpha) x)$; i.e., $x \theta x \neq 0$ for $\theta=\beta y \alpha$ in $\Gamma$. Thus $R$ is $N$-semisimple.

Conversely, let $R$ be $N$-semisimple and right Artinian. If possible, let $J(R) \neq 0$. Then $J(R)$ is nilpotent (by Proposition 2.11), so that $(J(R) \Gamma)^{n} J(R)=0$ and $I=(J(R) \Gamma)^{n-1} J(R) \neq 0$ for some integer $n$. Then $I \Gamma J(R)=0 ; I \Gamma I=0 ; x \Gamma x=0$ for $x \neq 0$ in $I$, a contradiction to $N$ semisimplicity. Thus $J(R)=0$ and $R$ is semisimple.

THEOREM 2.15. A $\Gamma$-ring is semisimple right Artinian if and only if it is isomorphic to a finite direct sum of simple right Artinian $\Gamma$-rings. 
Proof. Let $R$ be a semisimple right Artinian $\Gamma$-ring; then $0=$ $J(R)=\cap A(M)$, where $M$ runs over all irreducible $R \Gamma$-modules. $A(M)$ 's being right ideals of $R$, the right Artinian condition on $R$ implies the existence of a finite set $\left\{M_{1}, M_{2}, \cdots, M_{n}\right\}$ of irreducible $R \Gamma$-modules such that $\bigcap_{j=1}^{n} A\left(M_{j}\right)=0$ and $\bigcap_{j \neq i} A\left(M_{j}\right) \neq 0$ for each $i$ in $(1,2, \cdots, n)$. From Proposition 1.4, $R / A\left(M_{i}\right)$ will be primitive. Being a homomorphic image of $R$ it will also be right Artinian so that $R / A\left(M_{i}\right)$ will be a simple $\Gamma$-ring for each $i$ (by [10, Theorem 3.6]). Consider the map

$$
\begin{aligned}
\Theta: R & \longrightarrow \oplus \sum_{i=1}^{n} R / A\left(M_{i}\right) \\
x & \longrightarrow\left(x_{1}, x_{2}, \cdots, x_{n}\right), \text { where } x_{j}=x+A\left(M_{j}\right) .
\end{aligned}
$$

We claim that $\Theta$ is an isomorphism of $\Gamma$-rings. $\Theta$ is clearly a $\Gamma$ homomorphism; it is injective, in view of the fact that $\bigcap_{i=1}^{n} A\left(M_{i}\right)=0$. By the choice of $M_{1}, M_{2}, \cdots, M_{n}$, there exists an element $y^{k}(\neq 0)$ in $\bigcap_{i \neq k} A\left(M_{i}\right)$ such that $y^{k} \notin A\left(M_{k}\right)$. Therefore $y_{i}^{k}=0$ for $i \neq k$ and $y_{k}^{k} \neq 0$. Hence $\Theta\left(y^{k}\right)=\left(0,0, \cdots, y_{k}^{k}, 0, \cdots, 0\right)$. Since $R / A\left(M_{k}\right)$ is simple, $R / A\left(M_{k}\right)=\left\langle y_{k}^{k}\right\rangle$, where $\left\langle y_{k}^{k}\right\rangle$ is the ideal generated by $y_{k}^{k}$ in $R / A\left(M_{k}\right)$. By considering the elements of the ideal $\left\langle y_{k}^{k}\right\rangle$ one can easily conclude (as in $\left[12\right.$, p. 60]) that for any element $z_{k}$ in $R / A\left(M_{k}\right)$ there exists an $x$ in $R$ such that $\Theta(x)=\left(0,0, \cdots, 0, z_{k}, 0, \cdots, 0\right)$. Since $k$ is arbitrary in $(1,2, \cdots, n)$, it is clear that $\Theta$ is onto. Thus $R$ is isomorphic to the direct sum of the simple $\Gamma$-rings $R / A\left(M_{i}\right)$ which are also right Artinian. The converse part of the theorem is straightforward.

Part of the above proof was inspired by that of [12, Theorem 3.18].

Nobusawa has obtained earlier (see [15, Theorem 3]) a simultaneous decomposition for $R$ and $\Gamma$ under the stronger hypothesis of $R$ being a right and left Artinian $N$-semisimple $\Gamma_{N}$-ring. We also obtain below, a result with a similar but apparently stronger conclusion, using a possibly stronger hypothesis than that of Nobusawa. The choice of this hypothesis is anticipated by Corollary 3.5 proved later. For this we define a weak $\Gamma_{N}$-ring $R$ to be strongly semisimple (strongly simple, strongly right Artinian) if $R$ is semisimple (simple, right Artinian) as a weak $\Gamma_{N}$-ring and $\Gamma$ is semisimple (simple, right Artinian) as a weak $R_{N}$-ring. We then have

THEOREM 2.16. Let $R$ be a strongly semisimple strongly right Artinian weak $\Gamma_{N}$-ring. Then

$$
\begin{aligned}
& R=R_{1} \oplus R_{2} \oplus \cdots \oplus R_{n} \quad \text { and } \\
& \Gamma=\Gamma_{1} \oplus \Gamma_{2} \oplus \cdots \oplus \Gamma_{n},
\end{aligned}
$$


for ideals $R_{\text {r of }} R$ and $\Gamma_{i}$ of $\Gamma$, such that $R_{i}$ is a strongly simple, strongly right Artinian weak $\Gamma_{i N}-r i n g$ for $i=1,2, \cdots, n$.

Proof. By the previous result we can write $R=R_{1} \oplus R_{2} \oplus \cdots \oplus R_{n}$ and $\Gamma=\Gamma_{1} \oplus \Gamma_{2} \oplus \cdots \oplus \Gamma_{m}$ for simple $\Gamma$-rings $R_{i}$ and simple $R$ rings $\Gamma_{j}$. Since $R_{1} \Gamma R_{1} \neq 0, R_{1} \Gamma_{j} R_{1} \neq 0$ for at least one $j . R_{1} \Gamma_{j} R_{1}=$ $R_{1}$, in view of the simplicity of $R_{1}$. In view of the direct sum decomposition of $\Gamma$ it is clear that there can be only one $j=j_{1}$ with $R_{1} \Gamma_{j_{1}} R_{1} \neq 0$. For this $j_{1}, R_{1} \Gamma_{j_{1}} R_{1}=R_{1}$ so that $R_{1}=$ $R_{1} \Gamma_{j_{1}}\left(R_{1} \Gamma_{j_{1}} R_{1}\right)=R_{1}\left(\Gamma_{j_{1}} R_{1} \Gamma_{j_{1}}\right) R_{1} ; \quad \Gamma_{j_{1}} R_{1} \Gamma_{j_{1}} \neq 0 . \quad$ By simplicity of $\Gamma_{j_{1}}, \Gamma_{j_{1}} R_{1} \Gamma_{j_{1}}=\Gamma_{j_{1}}$. Thus, for each $i$ in $(1,2, \cdots, n)$ there exists a unique $j_{i}$ in $(1,2, \cdots, m)$ (necessarily distinct) such that $R_{i} \Gamma_{j_{i}} R_{\imath}=$ $R_{\imath}$ and $\Gamma_{j_{i}} R_{i} \Gamma_{j_{i}}=\Gamma_{j_{i}}$. Since we can reverse the role of $R$ and $\Gamma$, it is clear that $m=n$. By a rearrangement of the indices of $\Gamma_{j}$, one obtains the required decomposition. Rest of the assertions in the theorem are easily seen.

3. Jacobson radical for weak $\Gamma_{N}$-rings. In this section we obtain a few characterizations of the Jacobson radical valid for weak $\Gamma_{N}$-rings. Since any ring can be considered as a weak $\Gamma_{N}$-ring, our results in fact include some results on rings as well. We indicate this connection towards the end of this section.

Let $R$ be a weak $\Gamma_{N}$-ring and $\alpha$ be a fixed element in $\Gamma$. Then we denote by $R^{(\alpha)}$ the associative ring obtained by defining the composition $x \circ_{\alpha} y=x \alpha y$ in the additive group $R$. Clearly, a right ideal of $R$ is also a right ideal of the ring $R^{(\alpha)}$; further, an $x$ in $R$ is $\alpha$-rt. quasi-regular if and only if $x$ is right quasi-regular in the ring $R^{(\alpha)}$. In particular, the maximal $\alpha$-rt.q. $v^{\circ}$, right ideal $J_{\alpha}(R)$ of $R$ (see $\S 2$ ) is also a right quasi-regular right ideal of $R^{(\alpha)}$. In this setting we have

Theorem 3.1. Let $R$ be a weak $\Gamma_{N^{-r i n g}}$. Then $J(R)=\bigcap_{\alpha \in N} J^{*}\left(R^{(\alpha)}\right)$, where $J^{*}(\cdot)$ denotes the Jacobson radical of an associative ring.

Proof. From the remarks preceding the theorem it is clear that $J_{\alpha}(R) \subseteq J^{*}\left(R^{(\alpha)}\right)$ for each $\alpha$ in $\Gamma ; J(R) \subseteq \bigcap_{\alpha \in \Gamma} J^{*}\left(R^{(\alpha)}\right)$ (by Corollary 2.6). For proving the reverse inclusion we first recall Remark 2.4(ii) that $x$ in $R$ is $\alpha$-rt.q.r. if and only if the map $\Theta_{x, \alpha}: R \rightarrow R$ defined by $\Theta_{x, \alpha}(y)=y+x \alpha y$ is surjective. Let now $x \in \bigcap_{\alpha \in \Gamma} J^{*}\left(R^{(\alpha)}\right)$; then $\Theta_{x, \tilde{o}}$ is surjective for each $\delta$ in $\Gamma$. In particular, $\Theta_{x \beta y, \alpha}=\Theta_{x, \beta y \alpha}$ is surjective for all $\alpha, \beta$ in $\Gamma$ and $y$ in $R ; x \beta y$ is $\alpha-r t . q . r$. for each $\alpha$ in $\Gamma$. So $x \Gamma R$ is a right quasi-regular right ideal of $R$; therefore $x \in J(R)$, by Proposition 2.9. Thus $\bigcap_{\alpha \in,}, J^{*}\left(R^{(\alpha)}\right)=J(R)$. 
Let $A$ be any associative ring and $c$ be in $A$. Then the ring $A^{(c)}$ got by introducing the multiplication $a c b=a \circ b$ in the additive group $A$ is known as the $c$-homotope of $A$ (see [13, §2]). The $R^{(\alpha)}$ considered above is a somewhat similar structure. In fact, for $A$ considered as a weak $\Gamma_{N}$-ring, the two concepts are identical. Inspired by the associative case (see [13, Proposition 1]), we can call an element $x$ in a weak $\Gamma_{N}$-ring $R$ to be properly (right) quasi-invertible (p.q.i.) if $x$ is right quasi-regular in each $R^{(\alpha)}$. We then note that the p.q.i. elements are precisely the ones that we have already called as the right quasi-regular elements. As such, elements of $J(R)$ are p.q. invertible. On the other hand, if $x$ is $p . q . i$. in $R$, then $x$ is in $J(R)$ (as can be seen from the proof of the above Theorem 3.1). Thus we have

TheOREM 3.2. In a weak $\Gamma_{N}$-ring $R, J(R)$ is precisely the set of all p.q.i. elements of $R$.

We now note that the weak $\Gamma_{N}$-ring structure on $R$ enables us also to consider $\Gamma$ as a weak $R_{N}$-ring. As such, for an element $x$ in $R, \Gamma^{(x)}$ denotes the $x$-homotope of the weak $R_{N}$-ring $\Gamma$.

THEOREM 3.3. For a weak $\Gamma_{N}$-ring $R$,

$$
J(R)=\left\{x \in R \mid J^{*}\left(\Gamma^{(x)}\right)=\Gamma^{(x)}\right\} .
$$

Proof. Let $x$ be in $J(R)$. Then $x$ is $\beta$-quasi-regular for each $\beta$ in $\Gamma$. In particular, $x$ is $(-\alpha x \alpha)$-quasi-regular for each $\alpha$ in $\Gamma$. So, let $y$ be such that $x+y+x(-\alpha x \alpha) y=0$; hence $-\alpha x \alpha-\alpha y \alpha+$ $\alpha(x(\alpha x \alpha) y) \alpha=0$, i.e., $-\alpha x \alpha+(-\alpha y \alpha)+(-\alpha x \alpha) x(-\alpha y \alpha)=0$. This implies that $-\alpha x \alpha$ is an $x$-right quasi-regular element of $\Gamma$. Therefore, by Remark 2.4 (iv) $\alpha$ is $x$-right quasi-regular, i.e., $\alpha$ is right quasi-regular in the associative ring $\Gamma^{(x)} . \quad \alpha$ being arbitrary in $\Gamma$, clearly $J^{*}\left(\Gamma^{(x)}\right)=\Gamma^{(x)}$.

Conversely, let $x$ be in $R$ such that $J^{*}\left(\Gamma^{(x)}\right)=\Gamma^{(x)}$. Hence, every $\beta$ in $\Gamma$ is right quasi-regular in $\Gamma^{(x)}$. Therefore the map $\varphi_{\beta, x}: \Gamma \rightarrow \Gamma$ defined by $\varphi_{\beta, x}(\alpha)=\alpha+\beta x \alpha$ is surjective for each $\beta$ in $\Gamma$. So $\varphi_{-\alpha, x \alpha x}=\varphi_{-\alpha x \alpha, x}$ is surjective for each $\alpha$ in $\Gamma$. This implies that $-\alpha$ is $(x \alpha x)$-right quasi-regular. Let $\beta$ be in $\Gamma$ such that $-\alpha+$ $\beta-\alpha(x \alpha x) \beta=0$. This implies that $0=-x \alpha x+x \beta x-x(\alpha(x \alpha x) \beta) x=$ $-x \alpha x+x \beta x+(-x \alpha x) \alpha(x \beta x)$. Thus $(-x \alpha x)$ is $\alpha$-right quasi-regular; hence $x$ is $\alpha$-right quasi-regular (by Remark 2.4 (iv)). $\alpha$ being arbitrary in $\Gamma, x$ is p.q. invertible; therefore $x$ is in $J(R)$ (by Theorem $3.2)$. 
REMARK 3.4. Dualizing the above result we also have that $J(\Gamma)=\left\{\alpha \in \Gamma \mid J^{*}\left(R^{(\alpha)}\right)=R^{(\alpha)}\right\}$ in a weak $\Gamma_{N}$-ring $R$.

For later use we record the following immediate

CoRollary 3.5. If $R$ is a weak $\Gamma_{N}$-ring such that $\Gamma$ is semisimple, then $R$ is a $\Gamma_{N^{-}}$ing, i.e., $R \alpha R=0$ implies $\alpha=0$.

REMARK 3.6. Lister has developed in [8] a structure theory for the so-called ternary rings. These are easily seen to be also weak $\Gamma_{N}$-rings; besides, the notion of Jacobson radical for such a ternary ring $T$ coincides with that of the weak $\Gamma_{N}$-ring $T$ (see [8, Theorem 9]). As such, our earlier results hold for that case also. The radical theory there also is developed via a module theory.

For applying our results to the case of rings we first need the following result.

Theorem 3.7 (cf. [2, Theorem 10.1]). Let $J^{*}(A)$ be the Jacobson radical of a ring $A$ and $J(A)$ be the Jacobson radical of $A$ considered as a weak $\Gamma_{N}$-ring (see $\S 1$, Example 2). Then $J^{*}(A)=J(A)$.

Proof. $J(A)$ is clearly a right ideal of the ring $A$, by Proposition 2.9. Further, if $a$ is in $J(A)$, then $a$ is $(-a)$-quasi-regular in the $\Gamma$ ring $A$. Hence there exists an $a^{\prime}$ in $A$ such that $a+a^{\prime}-a a a^{\prime}=0$. One can then easily verify that $a$ is right quasi-regular in the ring $A$ with right quasi-inverse $-a-a a^{\prime}+a^{2} a^{\prime}$. Thus $J(A)$ is a right quasi-regular right ideal of the ring $A$ and hence is contained in $J^{*}(A)$.

Let, if possible, $J(A) \subsetneq J^{*}(A)$. Then there exists an irreducible $A \Gamma$-module $M$ such that $M \Gamma J^{*}(A) \neq 0$. Let $m$ in $M$ and $\alpha$ in $\Gamma(=A)$ be such that $m \alpha J^{*}(A) \neq 0$. By the irreducibility of $M, m \alpha J^{*}(A)=$ $M$; $m \alpha a=-m$ for some $a$ in $J^{*}(A)$. But $a \alpha \in J^{*}(A)$; hence $a \alpha+a^{\prime}+$ $a \alpha a^{\prime}=0$ for some $a^{\prime}$ in $A$. We have

$$
\begin{aligned}
0 & =m \alpha\left(\left(a \alpha+a^{\prime}+(a \alpha) a^{\prime}\right) a\right) \\
& =m \alpha\left(((\alpha \alpha) a)+a^{\prime} a+\left((a \alpha) a^{\prime}\right) a\right) \\
& =(m \alpha a) \alpha a+m \alpha\left(a^{\prime} a\right)+(m \alpha a) \alpha\left(a^{\prime} a\right) \\
& =m+m \alpha\left(a^{\prime} a\right)-m \alpha\left(a^{\prime} a\right)=m,
\end{aligned}
$$

a contradiction.

REMARK 3.8. One can also directly prove that the maximal regular right ideals of the ring $A$ are also maximal regular right 
ideals in the $\Gamma$-ring $A$ and vice versa. The above theorem can then be deduced also from this fact, using Proposition 2.3 and [4, Theorem 1.2.2].

Now we recall that an element $a$ in an associative ring $A$ is said to be properly quasi-invertible (p.q.i), if $a b$ is right quasi-regular in $A$ for each $b$ in $A$. It is clear that an element $a$ in $A$ is right quasi-regular if and only if the map $\Theta_{a}: A \rightarrow A$ defined by $\Theta_{a}(b)=$ $b+a b$ is surjective. Also, $a$ is right quasi-invertible in the $b$-homotope $A^{(b)}$ if and only if the map $\Theta_{a, b}: A \rightarrow A$ defined by $\Theta_{a, b}(c)=c+a b c$ is surjective. Since $\Theta_{a, b}=\Theta_{a b}$, the following known proposition is immediate.

Proposition 3.9 (cf. [13, Proposition 1]). An element $a$ in an associative ring $A$ is p.q.i. if and only if a is right quasi-invertible in each homotope $A^{(b)}$.

From the remarks preceding Theorem 3.2 and from Proposition 3.9 it is clear that an element $a$ in an associative ring $A$ is p.q.i. in $A$ if and only if it is p.q.i. in the $\Gamma$-ring $A$. Hence, in view of Theorem 3.7, the following corollary is a direct consequence of Theorems 3.1, 3.2, and 3.3.

Corollary 3.10. For an associative ring $A, J^{*}(A)$ is the set of all p.q.i. elements of $A$. Further,

$$
J^{*}(A)=\bigcap_{a \in A} J^{*}\left(A^{(a)}\right)=\left\{a \in A \mid J^{*}\left(A^{(a)}\right)=A^{(a)}\right\} .
$$

4. $\Gamma$-algebras, In this section we show how our results also apply to the case of ternary algebras and triple systems (see [11], (14]). For this we need the concept of a $\Gamma$-algebra which we define below.

Let $\Theta=\{\mathfrak{a}, \mathfrak{b}, \cdots\}$ be a commutative associative ring with identity and $\Gamma=\{\alpha, \beta, \cdots\}, E=\{x, y, \cdots\}$ be unitary right modules over $\Theta$. Then $E$ is said to be a $\Gamma$-algebra over $\Theta$, if $E$ is a $\Gamma$-ring satisfying $(x \alpha y) \mathfrak{a}=x \alpha(y \mathfrak{a})=x(\alpha \mathfrak{a}) y=(x \mathfrak{a}) \alpha y$. If further $E$ is also a weak $\Gamma_{N^{-}}$ ring we shall call $E$ to be a weak $\Gamma_{N^{-}}$algebra. It is clear that every $\Gamma$-ring $R$ can be considered as a $\Gamma$-algebra over the ring $Z$ of integers. Throughout this section $E$ will denote a $\Gamma$-algebra over a fixed ring $\Theta$ of scalars.

We shall call a $\Theta$-module $M$ to be an algebra module over $E$ (to be called an $E^{*} \Gamma$-module), if $M$ is an $E \Gamma$-module over the $\Gamma$-ring $E$ and if $(m \alpha x) \mathfrak{a}=m \alpha(x \mathfrak{a})=m(\alpha \mathfrak{a}) x=(m \mathfrak{a}) \alpha x$, for $m$ in $M, \alpha$ in $\Gamma$ and $\mathfrak{a}$ in $\Theta$. The notions of $E^{*} \Gamma$-submodules, algebra ideals etc. are 
self-evident. We shall further call an algebra right ideal $I$ of $E$ to be regular if it is regular as a $\Gamma$-ring ideal.

Proposition 4.1. A maximal regular algebra right ideal of $E$ is also a maximal regular ring right ideal of the $\Gamma$-ring $E$ and vice versa.

Proof. It clearly suffices to prove that a maximal regular ring right ideal $I$ of $E$ is also an algebra right ideal of $E$; in fact, it is enough to show that $I \Theta \subseteq I$. If $I \Theta \nsubseteq I$, then $I \Theta+I$ being also a $\Gamma$-ring right ideal, maximality of $I$ implies that $I+I \Theta=E$. By regularity of $I$ there exists an $e$ in $E$ and an $\alpha$ in $\Gamma$ such that $y-e \alpha y \in I$ for all $y$ in $E$. But $e \in I+I \Theta$; so, $e=x+\sum_{i=1}^{n} x_{i} a_{i}$, for $x, x_{i}$ in $I$ and $\mathfrak{a}_{i}$ in $\Theta(i=1,2, \cdots, n)$. Then $e \alpha e=x \alpha e+\Sigma\left(x_{i} \mathfrak{a}_{i}\right) \alpha e=$ $x \alpha e+\Sigma x_{i} \alpha\left(e a_{i}\right) \in I$. But $e-e \alpha e$ already belongs to $I$. So $e \in I$, a contradiction. Thus $I \Theta \subseteq I$.

The following proposition is a direct consequence of Proposition 4.1 if one observes that the irreducible $E \Gamma$-modules $\left(E^{*} \Gamma\right.$-modules) are precisely the modules of the form $E / I$ for maximal regular $\Gamma$ ring (algebra) right ideals $I$ of $E$ (see Lemma 1.3).

Proposition 4.2. Any irreducible $E^{*} \Gamma$-module is also irreducible as an EI-module. Conversely, any irreducible ET-module can be made into an $E^{*} \Gamma$-module.

As usual, we define the Jacobson radical $\bar{J}(E)$ of a $\Gamma$-algebra $E$ as $\cap A(M)$, where $M$ runs over all the irreducible $E^{*} \Gamma$-modules. Then, from Proposition 4.2 we immediately have

Proposition 4.3. Let $E$ be a $\Gamma$-algebra. Then $\bar{J}(E)=J(E)$.

REMARK 4.4. We note that all our earlier results could also have been obtained in the setting of algebras with suitable modifications. In particular, $\bar{J}(E)$ would also be the intersection of all maximal regular algebra right ideals of $E$ (Proposition 2.3).

We now recall that a ternary algebra $T$ over $\Theta$ is a unital (right) $\Theta$-module with a trilinear composition $(x, y, z) \rightarrow\langle x y z\rangle$ such that $\langle\langle x y z\rangle u v\rangle=\langle x\langle y z u\rangle v\rangle=\langle x y\langle z u v\rangle\rangle$ (see Myung [14]). Clearly, $T$ is also a weak $\Gamma_{N}$-algebra with $\Gamma=T$.

Now, let $T$ be a ternary algebra over $\Theta$. Then, by very definition, the notion of p.q. invertibility in the ternary algebra $T$ (see [14]) coincides with the same notion in the weak $\Gamma_{N}$-ring $T$ (see $\S 3$ ). Noting that the Jacobson radical $\operatorname{Rad} T$ of $T$ (as defined by Myung) 
consists precisely of the p.q.i. elements of $T$, we immediately have

Proposition 4.5. Let $T$ be a ternary algebra over $\Theta$. Then $\operatorname{Rad} T=\bar{J}(T)=J(T)$.

In view of the above proposition, the following (known) results (see [14, Theorem 3 and Corollary 1]) are direct consequences of Theorems 2.5, 3.1 and 3.3.

Proposition 4.6. Let $T$ be a ternary algebra over $\Theta$. Then $\operatorname{Rad} T=\bar{J}(T)$ is the unique maximal quasi-regular ideal in $T$. Further, $\operatorname{Rad} T=\bigcap_{x \in T} J^{*}\left(T^{(x)}\right)=\left\{x \in T \mid J^{*}\left(T^{(x)}\right)=T^{(x)}\right\}$.

REMARK 4.7. The above results show in particular that the concept of Jacobson radical for a ternary algebra could also have been developed in a natural way via a module theory for ternary algebras.

REMARK 4.8. The ternary algebras are also known in the literature (see [11], [14]) as associative triple systems of first kind. Indeed, an associative triple system of second kind is a unital $\Theta$-module $T$ together with a trilinear composition $\langle x y z\rangle$ such that $\langle\langle x y z\rangle u v\rangle=$ $\langle x\langle u z y\rangle v\rangle=\langle x y\langle z u v\rangle\rangle$. If $(T,\langle\cdot\rangle)$ is an associative triple system of the second kind, then $T$ also becomes a weak $\Gamma_{N}$-algebra, if one defines the ternary compositions as follows: $\Gamma=T$ and the $\Gamma$-ring composition in $T$ be same as $\langle\cdot\rangle$; the weak $\Gamma_{N}$-ring composition in $\Gamma$ is defined by $(\alpha x \beta)=\langle\beta x \alpha\rangle$. Also, the Jacobson radical of $T$ (as defined in [11]) coincides with $J(T)$ as defined in the present paper. Thus, our set-up provides a uniform module cum radical theory for the triple systems of both kinds at the same time.

REMARK 4.9. Hestenes considers in [5] a special type of (finite dimensional) ternary algebra over complex numbers. This algebra also becomes a weak $\Gamma_{N}$-ring in a natural way and in fact it is also semisimple in the sense of Nobusawa (see Condition 4 in Hestenes' definition). As such, some of his results can also be deduced from ours.

REMARK 4.10. Stephenson considers in [16] certain ternary rings, earlier introduced by Hestenes; such ternary rings, by very definition, are associative triple systems of second kind. Stephenson raises certain questions concerning the Jacobson radical for such systems. We note here that almost all his querries find their answers in the preceding material.

5. Imbedding of a weak $\Gamma_{N}$-ring into an associative ring. In 
this section we describe a construction by which a weak $\Gamma_{N}$-ring can be imbedded into an associative ring with identity. This construction is similar to the one given in [11] for associative triple systems.

Let $R$ be a weak $\Gamma_{N}$-ring. For $x$ in $R$ and $\alpha$ in $\Gamma$, let $L(x, \alpha)$, $L(\alpha, x), R(x, \alpha)$, and $R(\alpha, x)$ respectively denote the mappings $y \rightarrow x \alpha y$, $\beta \rightarrow \alpha x \beta, \beta \rightarrow \beta x \alpha$ and $y \rightarrow y \alpha x$. Clearly, $L(x, \alpha)$ and $R(\alpha, x) \in$ End $R, R(x, \alpha)$ and $L(\alpha, x) \in$ End $\Gamma$. Now consider the ring $E=$ End $R \oplus(\text { End } \Gamma)^{o p}$. For $x$ in $R$ and $\alpha$ in $\Gamma$, let $l(x, \alpha)$ denote the element $(L(x, \alpha), R(x, \alpha))$ in $E$. We denote by $L$ the subring of $E$ generated by $\{l(x, \alpha)\}$ for $x$ in $R$ and $\alpha$ in $\Gamma$. The multiplication in $E$ shows that $l(x, \alpha) l(y, \beta)=l(x \alpha y, \beta)=l(x, \alpha y \beta)$. In a similar way one can associate with $\alpha$ in $\Gamma$ and $x$ in $R$ the element $r(\alpha, x)=$ $(R(\alpha, x), L(\alpha, x))$ in (End $R)^{o p} \oplus$ End $\Gamma=E^{o p}$. Again, let $S$ denote the subring of $E^{o p}$ generated by $\{r(\alpha, x)\}$ for $\alpha$ in $\Gamma$ and $x$ in $R$. Multiplication in $E^{o p}$ shows that $r(\alpha, x) r(\beta, y)=r(\alpha x \beta, y)=r(\alpha, x \beta y)$. Let $E_{1}$ and $E_{2}$ respectively denote the identity elements of $E$ and $E^{o p}$. We set $\mathscr{L}=Z E_{1}+L$ and $\mathscr{R}=Z E_{2}+S, Z$ denoting the set of integers. $R$ and $\Gamma$ respectively become $E-E^{o p}$ and $E^{o p}-E$ bimodules with respect to the following module multiplications: For $(P, \Theta)$ in $E,\left(P_{1}, \Theta_{1}\right)$ in $E^{o p}\left(P, P_{1}\right.$ in End $R$ and $\Theta, \Theta_{1}$ in $\left.\operatorname{End} \Gamma\right), x$ in $R$ and $\alpha$ in $\Gamma,(P, \Theta) x=P(x), x\left(P_{1}, \Theta_{1}\right)=P_{1}(x), \alpha(P, \Theta)=\Theta(\alpha)$ and $\left(P_{1}, \Theta_{1}\right) \alpha=\Theta_{1}(\alpha)$. Consequently, $R$ and $\Gamma$ can also be considered respectively as $\mathscr{L}-\mathscr{R}$ and $\mathscr{R}-\mathscr{L}$ bimodules. We shall use these module multiplications in the sequel without explicit mention.

Now we consider the sum $A=\mathscr{L} \oplus R \oplus \Gamma \oplus \mathscr{R}$, where $\oplus$ denotes (additive) group direct sum. Denoting the elements of $A$ by $\left(\begin{array}{ll}l & x \\ \alpha & r\end{array}\right)$ with $l$ in $\mathscr{L}, x$ in $R, \alpha$ in $\Gamma$ and $r$ in $\mathscr{R}$, we introduce a multiplication in $A$ by defining

$$
\left(\begin{array}{ll}
l_{1} & x_{1} \\
\alpha_{1} & r_{1}
\end{array}\right)\left(\begin{array}{ll}
l_{2} & x_{2} \\
\alpha_{2} & r_{2}
\end{array}\right)=\left(\begin{array}{ll}
l_{1} l_{2}+l\left(x_{1}, \alpha_{2}\right) & l_{1} x_{2}+x_{1} r_{2} \\
\alpha_{1} l_{2}+r_{1} \alpha_{2} & r\left(\alpha_{1}, x_{2}\right)+r_{1} r_{2}
\end{array}\right) .
$$

Then one can easily verify that $A$ is an associative ring with respect to this multiplication with identity element $I d=\left(\begin{array}{ll}E_{1} & 0 \\ 0 & E_{2}\end{array}\right)$. Further, the elements $x, \alpha, l$ and $r$ of $R, \Gamma, \mathscr{L}$ and $\mathscr{R}$ can be respectively identified with the elements $\left(\begin{array}{ll}0 & x \\ 0 & 0\end{array}\right),\left(\begin{array}{ll}0 & 0 \\ \alpha & 0\end{array}\right),\left(\begin{array}{ll}l & 0 \\ 0 & 0\end{array}\right)$ and $\left(\begin{array}{ll}0 & 0 \\ 0 & r\end{array}\right)$ of $A$. The weak $\Gamma_{N}$-ring multiplications are preserved by this identification; so we can assume that $R$ and $\Gamma$ are imbedded in $A$ in the above manner. From the definition of multiplication in $A$ the following results are easily deduced.

Proposition 5.1. $A_{0}=L \oplus R \oplus \Gamma \oplus S$ is an ideal of $A$. 
Proposition 5.2 (cf. [11], Theorem 2 (v), p. 30]). $E_{1}$ is an idempotent in $A$ and the Peirce spaces of $A$ relative to $E_{1}$ are given by: $A_{11}=\mathscr{L}, A_{10}=R, A_{01}=\Gamma$ and $A_{00}=\mathscr{R}$.

REMARK 5.3. If $R$ is a weak $\Gamma_{N}$-ring, then $\Gamma$ is also a weak $R_{N}$-ring. As such, we could have as well started with $\Gamma$ and obtained, through the above construction, another associative ring $A^{\prime}=\mathscr{L}_{\Gamma} \oplus$ $\Gamma \oplus R \oplus \mathscr{R}_{\Gamma}$. However, one can easily see that there is a natural isomorphism between $A$ and $A^{\prime}$ which preserves the structure of the components individually; thus, this second construction does not yield any new structure.

Proposition 5.4. If $I$ is an ideal of $A$, then

(i ) $I=\bigoplus \sum_{i=0,1} \sum_{j=0,1}\left(I \cap A_{i j}\right)$.

(ii) $I \cap R$ and $I \cap \Gamma$ are ideals of $R$ and $\Gamma$ respectively.

(iii) If $I \subseteq \mathscr{L} \oplus \mathscr{R}$, then $I=0$.

Proof. (i) and (ii) are clear (see [11, Lemma 7, p. 18]). For (iii), let $I \subseteq \mathscr{L} \oplus \mathscr{R} . \quad$ By (i) and Proposition 5.2, $I=(I \cap \mathscr{L}) \oplus(I \cap R) \oplus$ $(I \cap \Gamma) \oplus(I \cap \mathscr{R})$; by hypothesis, $I \cap R=0$ and $I \cap \Gamma=0$; hence $I=(I \cap \mathscr{L}) \oplus(I \cap \mathscr{R})$. Also $(I \cap \mathscr{L}) R \cong(I R) \cap(\mathscr{L} R) \subseteq I \cap R=0$ and $\Gamma(I \cap \mathscr{L})=(\Gamma I) \cap(\Gamma \mathscr{L}) \subseteq I \cap \Gamma=0$; therefore $I \cap \mathscr{L}=0$; similarly $I \cap \mathscr{R}=0$. Thus $I=0$.

For a $\Gamma_{N}$-ring $R$, the rings $L$ and $S$ can be identified in a natural way with the left and right operator rings of $R$ (for definitions see [10]). To see this we need the following

Lemma 5.5. Let $R$ be a $\Gamma_{N}$-ring. Then, for an $l$ in $L(r$ in $S)$ $l y=0(y r=0)$ for all $y$ in $R$ if and only if $1=0(r=0)$.

Proof. Let $l=\sum_{i=1}^{n} 1\left(x_{i}, \alpha_{i}\right)=\left(\Sigma L\left(x_{i}, \alpha_{i}\right), \Sigma R\left(x_{i}, \alpha_{i}\right)\right)$ be in $L$ such that $l y=0$ for all $y$ in $R$; hence $\Sigma L\left(x_{i}, \alpha_{i}\right) y=0$ for all $y$ in $R$, i.e., $\Sigma L\left(x_{i}, \alpha_{i}\right)=0$ in End $R$. Further, $x\left(\Sigma \beta x_{i} \alpha_{i}\right) y=x \beta\left(\Sigma x_{i} \alpha_{i} y\right)=0$ for any $x, y$ in $R$ and $\beta$ in $\Gamma$. Hence, by the Nobusawa condition, $0=\Sigma \beta x_{i} \alpha_{i}=\beta \Sigma R\left(x_{i}, \alpha_{i}\right)=\Sigma R\left(x_{i}, \alpha_{i}\right)(\beta)$ for all $\beta$ in $\Gamma$, i.e., $\Sigma R\left(x_{i}, \alpha_{i}\right)=$ 0 in End $\Gamma$. Thus $l=0$. The other part is similar.

Proposition 5.6. Let $R$ be a $\Gamma_{N}$-ring and $0_{l t}^{R}, 0_{r t}^{R}$ respectively denote the left and right operator rings of $R$ (see [10]). Then $0_{l t}^{R} \cong L$ and $0_{r t}^{R} \cong S$.

Proof. The map $\varphi: 0_{1 t}^{R} \rightarrow L$ defined by $\varphi\left(\sum_{i=1}^{n}\left[x_{i}, \alpha_{i}\right]\right)=\sum_{i=1}^{n} l\left(x_{i}, \alpha_{i}\right)$ is well-defined in view of the above lemma. Further, it can be 
easily seen to be a ring epimorphism; that ker $\phi=0$ follows from the definition of left operator ring. Thus $\varphi$ is the required isomorphism. The other part is similar.

The following result can be verified on the same lines as the above proposition; it is also implicit in the proof of Lemma 5.5.

Proposition 5.7. Let $R$ be a $\Gamma_{N}$-ring and $\Gamma$ be an $R_{N}$-ring. Then $0_{r t}^{R} \cong 0_{l t}^{R}$ and $0_{l t}^{\Gamma} \cong 0_{r t}^{\Gamma}$.

6. Strongly semisimple weak $\Gamma_{N}$-rings. In this section we shall show the equivalence of the semisimplicity (and minimum condition) of $A$ and strong semisimplicity (and strong Artinian condition) of $R$. Towards this we first have

Proposition 6.1. An element $x$ in a weak $\Gamma_{N}$-ring $R$ is p.q. invertible (see §3) in $R$ if and only if it is p.q. invertible in the associative ring $A$.

Proof. Let $x$ be p.q. invertible in the weak $\Gamma_{N}$-ring $R=A_{10}=$ $E_{1} A\left(I d-E_{1}\right)$. Hence $x$ is right quasi-regular in $R^{(\alpha)}$, for each $\alpha$ in $\Gamma=A_{01}=\left(I d-E_{1}\right) A E_{1}$; thus, for each $a$ in $A$ there exists a $y$ in $R$ such that $0=x+y+x\left(\left(I d-E_{1}\right) a E_{1}\right) y=x+y+\left(x\left(I d-E_{1}\right)\right) a\left(E_{1} y\right)$. Since $x, y$ are in $A_{10}$ this implies that $x+y+x a y=0$; hence $x$ is right quasi-regular in each homotope $A^{(a)}$, i.e., $x$ is p.q. invertible in $A$.

Conversely, let $x$ in $R$ be right quasi-regular in $A^{(a)}$ for each $a$ in $A$; in particular, $x$ is right quasi-regular in $A^{(\alpha)}$ for each $\alpha$ in $\Gamma$. If $y=y_{11}+y_{10}+y_{01}+y_{00}$ (with $y_{i j}$ in $A_{i j}, i, j=0,1$ ) be the right quasi-inverse of $x$ in $A^{(\alpha)}$, then one can see (using the multiplication rules among the $A_{i j}$ 's) that $y_{10}$ is a right quasi-inverse of $x$ in $R^{(\alpha)}$. Hence $x$ is $p . q$. invertible in $R$.

By a similar reasoning as above, we have

Proposition 6.2. Let $R$ be a weak $\Gamma_{N}$-ring. Then an $\alpha$ in $\Gamma$ is p.q.i. in $\Gamma$ if and only if $\alpha$ is p.q.i. in $A$.

The following corollary is immediate from Propositions 6.1, 6.2 in view of Theorem 3.2 and Corollary 3.10.

COROLlaRY 6.3. Let $R$ be a weak $\Gamma_{N}$-ring and let $J(R), J(\Gamma)$ and $J^{*}(A)$ respectively denote the Jacobson radicals of $R, \Gamma$ and $A$. Then $J(R)=R \cap J^{*}(A)$ and $J(\Gamma)=\Gamma \cap J^{*}(A)$. 
By Proposition 5.4(i) we have $J^{*}(A)=\left(J^{*}(A) \cap \mathscr{L}\right) \oplus\left(J^{*}(A) \cap R\right) \oplus$ $\left(J^{*}(A) \cap \Gamma\right) \oplus\left(J^{*}(A) \cap \mathscr{R}\right)$. We then use Corollary 6.3 and [11, Theorem 5 (ii), p. 18] to obtain

LeMmA 6.4. $J^{*}(A)=J^{*}(\mathscr{L}) \oplus J(R) \oplus J(\Gamma) \oplus J^{*}(\mathscr{R})$.

THEOREM 6.5. $R$ is strongly semisimple if and only if $A$ is semisimple.

Proof. If $A$ is semisimple then $R$ is strongly semisimple, by Lemma 6.4. Conversely, if $R$ is strongly semisimple then $J^{*}(A)=$ $J^{*}(\mathscr{L}) \oplus J^{*}(\mathscr{R}) \subseteq \mathscr{L} \oplus \mathscr{R}$. Since $J^{*}(A)$ is an ideal of $A$, it follows from Proposition 5.4 (iii) that $J^{*}(A)=0$; hence $A$ is semisimple.

If $I_{1} \supset I_{2} \supset \cdots$ is a strictly descending chain of right ideals of $R$, then one can easily see that $l\left(I_{1}, \Gamma\right)+I_{1} \supset l\left(I_{2}, \Gamma\right)+I_{2} \supset \cdots$ is a strictly descending chain of right ideals of $A$. As such, if $A$ is right Artinian, then $R$ (and similarly $\Gamma$ ) is also right Artinian, i.e., $R$ is strongly right Artinian. The converse however needs certain extra assumptions. Towards that we first have

Lemma 6.6. If $R$ is a strongly right Artinian, strongly semisimple weak $\Gamma_{N}$-ring, then $L$ and $S$ are also semisimple and right Artinian.

Proof. By Theorem 2.14, $R$ and $\Gamma$ are semisimple in the sense of Nobusawa. As such we can write (see [15, Theorem 1]) $R=$ $e_{1} 0_{r t}^{R} \oplus \cdots \oplus e_{n} 0_{r t}^{R}$ and $\Gamma=\alpha_{1} 0_{r t}^{\Gamma} \oplus \cdots \oplus \alpha_{m} 0_{r t}^{\Gamma}$ for minimal right ideals $e_{i} 0_{r t}^{R}\left(\alpha_{j} 0_{r t}^{\Gamma}\right)$ of $R(\Gamma)$. One can then easily verify that

$$
0_{r t}^{R}=\sum_{i=1}^{n} \sum_{j=1}^{m}\left[\alpha_{j}, e_{i}\right] 0_{r t}^{R}
$$

and that $\left[\alpha_{j}, e_{i}\right] 0_{r t}^{R}$ is a minimal right ideal of $0_{r t}^{R}$ for all $i, j .0_{r t}^{R}$ will be a finite direct sum of minimal right ideals of $0_{r t}^{R}$ (see [7, Lemma on p. 442]). Then, $0_{r t}^{R}$ being an operator group with a composition series will be right Artinian. In a similar way we can show that $0_{r t}^{r}$ is also right Artinian. Since $R$ is a $\Gamma_{N}$-ring and $\Gamma$ an $R_{N}$-ring (by Corollary 3.5) we can use Propositions 5.6 and 5.7 to conclude that $L$ and $S$ are right Artinian. $L$ and $S$ are semisimple, being ideals of the semisimple rings $\mathscr{S}$ and $\mathscr{R}$ respectively (by using Theorem 6.5 and Lemma 6.4).

LEMMA 6.7. If $R$ is strongly right Artinian and strongly semisimple, then $L=\mathscr{L}, S=\mathscr{R}$ and hence $A_{0}=A$. 
Proof. From the previous lemma and from [3, Corollary 1, p. 32] it follows that $L$ and $S$ have identity elements. Let $l=\Sigma l\left(x_{i}, \alpha_{i}\right)=$ $\Sigma\left(L\left(x_{i}, \alpha_{i}\right), R\left(x_{i}, \alpha_{i}\right)\right)$ be the identity element of $L$; then $l$ is the identity element of $E=\operatorname{End} R \oplus(\text { End } \Gamma)^{o p}$. If not, either $\Sigma L\left(x_{i}, \alpha_{i}\right)(y) \neq y$ for some $y$ in $R$ or $\Sigma R\left(x_{i}, \alpha_{i}\right)(\beta) \neq \beta$ for some $\beta$ in $\Gamma$. For example, if $\Sigma x_{i} \alpha_{i} y-y \neq 0$, then by Corollary 3.5 there exist $\beta$ and $\partial$ in $\Gamma$ such that $0 \neq \beta\left(\Sigma x_{i} \alpha_{i} y-y\right) \partial=\beta\left(\left(\Sigma l\left(x_{i}, \alpha_{i}\right)\right) l(y, \partial)\right)-\beta l(y, \partial)=$ $\beta l(y, \partial)-\beta l(y, \partial)=0$, a contradiction. Thus $l=E_{1}$ and hence $L=$ $\mathscr{L}$. Similarly $S=\mathscr{R}$.

Theorem 6.8. Let $R$ be a weak $\Gamma_{N^{-}}$.ring. Then $R$ is strongly semisimple strongly right Artinian if and only if $A$ is semisimple and right Artinian.

Proof. It only remains to prove that $A$ is right Artinian when $R$ is strongly semisimple and strongly right Artinian. Using Lemma 6.7 we can write $A=A E_{2} \oplus A E_{1}=(R \oplus S) \oplus(\Gamma \oplus L)$. Further, any right ideal $I$ of $A$ can be written as $I=I E_{2} \oplus I E_{1}$, where $I E_{2}\left(I E_{1}\right)$ is a right ideal of the ring $A E_{2}\left(A E_{1}\right)$. In view of this it is enough to show that $A E_{2}$ and $A E_{1}$ satisfy right Artinian conditions. Since $R$ and $S$ are semisimple and right Artinian (by Lemma 6.6) we have $R=e_{1} S \oplus \cdots \oplus e_{n} S$ and $S=f_{1} S \oplus \cdots \oplus f_{m} S$ with $e_{i}$ 's in $R$ and $f_{j}^{\prime}$ 's in $S$ such that $e_{i} S$ and $f_{j} S$ are minimal right ideals of the weak $\Gamma_{N}$-ring $R$ and the ring $S$ respectively. Then $A E_{2}=\Sigma e_{i} S \oplus \Sigma f_{j} S=$ $\Sigma e_{i} A E_{2} \oplus \Sigma f_{j} A E_{2} . \quad e_{2} A E_{2}$ and $f_{j} A E_{2}$ are easily seen to be minimal right ideals of $A E_{2}$. As before (see the proof of Lemma 6.6) $A E_{2}$ is seen to be right Artinian. Similarly $A E_{1}$ will be right Artinian; hence $A$ is right Artinian.

The following corollary is immediate from the above theorem if one recalls that a semisimple ring is right Artinian if and only if it is left Artinian (see [6]).

Corollary 6.9. Let $R$ be a strongly semisimple weak $\Gamma_{N}$-ring. Then $R$ is strongly right Artinian if and only if $R$ is strongly left Artinian.

7. Strongly simple $\Gamma$-rings. In this section we characterize completely the strongly simple strongly right Artinian weak $\Gamma_{N}$-rings.

Proposition 7.1. If $A$ is simple then $R$ is strongly simple.

Proof. It suffices to observe that for a nonzero proper ideal $I\left(\Gamma_{1}\right)$ of $R(\Gamma), A I A\left(A \Gamma_{1} A\right)$ is a proper ideal of $A$ containing $I\left(\Gamma_{1}\right)$. 


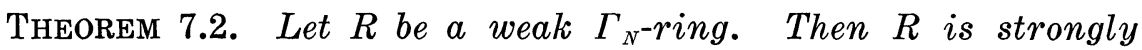
simple strongly right Artinian if and only if $A$ is simple and right Artinian.

Proof. Let $R$ be strongly simple and strongly right Artinian. Then $R$ is also strongly semisimple, so that $A$ is right Artinian (by Theorem 6.8). If $I$ be a nonzero ideal of $A$ then $I \cap R(I \cap \Gamma)$ is an ideal of $R(\Gamma)$. Strong simplicity of $R$ implies that $I \cap R=0$ or $R$ and that $I \cap \Gamma=0$ or $\Gamma$. If $I \cap R=0$ and $I \cap \Gamma=0$, then by Proposition 5.4 (i) and (iii) $I=0$, a contradiction. If however $I \cap R=R$, then $\Gamma=\Gamma R \Gamma=\Gamma(I \cap R) \Gamma \leqq I$ so that $I \cap \Gamma=\Gamma$. Therefore $I \supseteqq$ $R \oplus \Gamma$. Since $I$ is an ideal of $A, I \supseteqq \Gamma R=S$ and $I \supseteqq R \Gamma=L$. Therefore $I \supseteqq L \oplus R \oplus \Gamma \oplus S=A_{0}$. By Lemma 6.7, $I=A_{0}=A$. Hence $A$ is also simple. The converse is immediate from Proposition 7.1 and Theorem 6.8.

THEOREM 7.3. Let $R$ be a strongly simple strongly right Artinian weak $\Gamma_{N}$-ring. Then there exists a division ring $D$ and positive integers $m, n$ such that $R \cong M_{n},{ }_{m}(D)$ and $\Gamma \cong M_{m, n}(D)$ with the weak $\Gamma_{N}$-ring multiplications being the ordinary matrix multiplications (see $\S 1$, Example 1(b)).

Proof. By Theorem 7.2, $A$ is simple and right Artinian; hence $A$ is isomorphic to a matrix ring of the form $M_{k, k}(D)$, for some division ring $D$ (see [6]). Without loss of generality we can assume that the idempotent $E_{1}$ in $A$ has the following matrix representation with respect to the above identification of $A$ with $M_{k, k}(D)$.

$$
E_{1}=\left(\begin{array}{l|l}
I_{n, n} & 0_{n, m} \\
\hline 0_{m, n} & 0_{m, m}
\end{array}\right),
$$

$m, n$ being integers with $m+n=k$. The element $E_{2}=I d-E_{1}$ will consequently have the representation

$$
\left(\begin{array}{l|l}
0_{n, n} & 0_{n, m} \\
\hline 0_{m, n} & I_{m, m}
\end{array}\right) .
$$

By appealing to Proposition 5.2 we have the following further identifications of the components of $A$ with subsets of $M_{k, k}(D)$.

$$
\begin{aligned}
R=E_{1} A\left(I d-E_{1}\right) & =\left(\begin{array}{c|c}
I_{n, n} & 0_{n, m} \\
\hline 0_{m, n} & 0_{m, m}
\end{array}\right) M_{k, k}(D)\left(\begin{array}{c|c}
0_{n, n} & 0_{n, m} \\
\hline 0_{m, n} & I_{m, m}
\end{array}\right) \\
& =\left(\begin{array}{c|c}
0_{n, n} & M_{n, m}(D) \\
\hline 0_{m, n} & 0_{m, m}
\end{array}\right) .
\end{aligned}
$$




$$
\begin{aligned}
\Gamma=\left(I d-E_{1}\right) A E_{1} & =\left(\begin{array}{c|c}
0_{n, n} & 0_{n, m} \\
\hline 0_{m, n} & I_{m, m}
\end{array}\right) M_{k, k}(D)\left(\begin{array}{c|c}
I_{n, n} & 0_{n, m} \\
\hline 0_{m, n} & 0_{m, m}
\end{array}\right) \\
& =\left(\begin{array}{c|c}
0_{n, n} & 0_{n, m} \\
\hline M_{m, n}(D) & 0_{m, m}
\end{array}\right) .
\end{aligned}
$$

The conclusions of the theorem clearly follow from the above representations of $R$ and $\Gamma$.

REMARK 7.4. Under the hypothesis of the above theorem we can also identify the left operator ring $0_{l t}^{R}$ of $R$ with $M_{n, n}(D)$, a complete matrix ring, and the right operator ring $0_{r t}^{R}$ with the complete matrix ring $M_{m, m}(D)$ (as in the above theorem, using Proposition 5.6, Corollary 3.5 and Lemma 6.7).

REMARK 7.5. A ternary ring $T$ in the sense of Lister (see [8]) is also a weak $\Gamma_{N}$-ring with $\Gamma=T$ (see Remark 3.6). An ideal of $T$ is defined as a two-sided ideal which is also medial. However in the case of a weak $\Gamma_{N}$-ring the notion of a medial ideal deoes not exist. As such, a ternary ring $T$ which is simple as a weak $\Gamma_{N}$-ring is also simple as a ternary ring. One can easily show by example (see [8]) that a simple ternary ring need not be simple as a weak $\Gamma_{N}$-ring.

REMARK 7.6. Recalling that a Hestenes ternary algebra is also a semisimple weak $\Gamma_{N}$-ring (see Remark 4.9) we note that the main Theorem 6.6 of [5] has a bearing to our Theorem 7.3.

Professor J. Luh has kindly communicated to us a weaker form of the following result:

If $R$ is strongly semisimple then $R$ is strongly right Artinian if and only if $R$ is right and left Artinian. One way (only if part) follows from Corollary 6.9. The other part can be deduced from Nobusawa's structure theorems (see [15]) using Theorem 2.14. Professor Luh's proof for the first part of the particular case is different from ours.

\section{REFERENCES}

1. W. E. Barnes, On the $\Gamma$-rings of Nobusawa, Pacific J. Math., 18 (1966), 411-422.

2. W. E. Coppage and J. Luh, Radicals of $\Gamma$-rings, J. Math. Soc. Japan, 23 (1971), $40-52$.

3. M. Gray, A radical Approach to Algebra, Addison-Wesley, 1970.

4. I. N. Herstein, Non-commutative rings, Carus Math. monograph No. 15 (1969).

5. M. R. Hestenes, On a ternary algebra, Scripta Mathematica, Volume XXIX, No. 3-4, 253-272 (1973). 
6. I. Kaplansky, Fields and Rings, Univ. of Chicago Press, 1972.

7. S. Lang, Algebra, Addison-Wesley, 1965.

8. W. G. Lister, Ternary rings, Trans. Amer. Math. Soc., 154 (1971), 37-55.

9. J. Luh, On primitive $\Gamma$-rings with minimal one-sided ideals, Osaka J. Math., 5 (1968), 165-173.

10. - On the theory of simple $\Gamma$-rings, Michigan Math. J., 16 (1969), 65-75.

11. K. Meyberg, Lectures on algebras and triple systems, The University of Virginia, Charlottesville, 1972.

12. N. H. McCoy, Ring Theory, Macmillan, 1966.

13. K. McCrimmon, A characterization of the radical of a Jordan algebra, J. Algebra, 18 (1971), 103-111.

14. H. C. Myung, A characterization of Jacobson radical in ternary algebra, Proc. Amer. Math. Soc., 38 (1973), 228-234.

15. N. Nobusawa, On a generalization of ring theory, Osaka J. Math., 1 (1964), 81-89.

16. R. A. Stephenson, Jacobson structure theory for Hestenes' ternary rings, Trans. Amer. Math. Soc., 177 (1973), 91-98.

Received November 26, 1975.

Birla Institute of Technology and Science

Pilani 333031 (Rajasthan) India

AND

Nehru COLLEge

FARRUKHABAD (U.P.) INDIA 



\section{PACIFIC JOURNAL OF MATHEMATICS}

\section{EDITORS}

RICHARD ARENS (Managing Editor)

University of California

Los Angeles, CA 90024

Charles W. Curtis

University of Oregon

Eugene, OR 97403

C. C. MOORE

University of California

Berkeley, CA 94720
J. DUGUNDJI

Department of Mathematics

University of Southern California

Los Angeles, CA 90007

R. FINN and J. MILGRAM

Stanford University

Stanford, CA 94305

\section{ASSOCIATE EDITORS}

\section{E. F. BeCKENBACH}

B. H. NeumanN

F. WOLF

K. YoSHIDA

\section{SUPPORTING INSTITUTIONS}

\author{
UNIVERSITY OF SOUTHERN CALIFORNIA \\ STANFORD UNIVERSITY \\ UNIVERSITY OF HAWAII \\ UNIVERSITY OF TOKYO \\ UNIVERSITY OF UTAH \\ WASHINGTON STATE UNIVERSITY \\ UNIVERSITY OF WASHINGTON
}

The Supporting Institutions listed above contribute to the cost of publication of this Journal, but they are not owners or publishers and have no responsibility for its content or policies.

Mathematical papers intended for publication in the Pacific Journal of Mathematics should be in typed form or offset-reproduced, (not dittoed), double spaced with large margins. Please do not use built up fractions in the text of the manuscript. However, you may use them in the displayed equations. Underline Greek letters in red, German in green, and script in blue. The first paragraph or two must be capable of being used separately as a synopsis of the entire paper. Items of the bibliography should not be cited there unless absolutely necessary, in which case they must be identified by author and journal, rather than by item number. Manuscripts, in triplicate, may be sent to any one of the editors. Please classify according to the scheme of Math. Reviews, Index to Vol. 39. All other communications should be addressed to the managing editor, or Elaine Barth, University of California, Los Angeles, California, 90024.

50 reprints to each author are provided free for each article, only if page charges have been substantially paid. Additional copies may be obtained at cost in multiples of 50 .

The Pacific Journal of Mathematics is issued monthly as of January 1966. Regular subscription rate: $\$ 72.00$ a year (6 Vols., 12 issues). Special rate: $\$ 36.00$ a year to individual members of supporting institutions.

Subscriptions, orders for numbers issued in the last three calendar years, and changes of address should be sent to Pacific Journal of Mathematics, P.O. Box 969, Carmel Valley, CA 93924, U.S.A. Older back numbers obtainable from Kraus Periodicals Co., Route 100, Millwood, NY 10546.

PUBLISHED BY PACIFIC JOURNAL OF MATHEMATICS, A NON-PROFIT CORPORATION

Printed at Kokusai Bunken Insatsusha (International Academic Printing Co., Ltd.). 8-8, 3-chome, Takadanobaba, Shinjuku-ku, Tokyo 160, Japan. 


\section{Pacific Journal of Mathematics}

\section{Vol. 80, No. $2 \quad$ October, 1979}

K. Adachi, On the multiplicative Cousin problems for $N^{p}(D) \ldots \ldots \ldots \ldots 297$

Howard Banilower, Isomorphisms and simultaneous extensions in $C(S) \ldots 305$

B. R. Bhonsle and R. A. Prabhu, An inversion formula for a distributional

finite-Hankel-Laplace transformation ................... 313

Douglas S. Bridges, Connectivity properties of metric spaces.......... 325

John Patton Burgess, A selection theorem for group actions ........... 333

Carl Claudius Cowen, Commutants and the operator equations

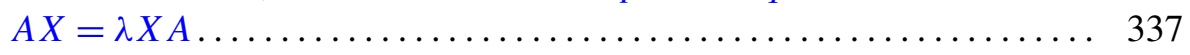

Thomas Curtis Craven, Characterizing reduced Witt rings. II .......... 341

J. Csima, Embedding partial idempotent d-ary quasigroups ............ 351

Sheldon Davis, A cushioning-type weak covering property ............ 359

Micheal Neal Dyer, Nonminimal roots in homotopy trees ............. 371

John Erik Fornaess, Plurisubharmonic defining functions ........... 381

John Fuelberth and James J. Kuzmanovich, On the structure of finitely

generated splitting rings .......................... 389

Irving Leonard Glicksberg, Boundary continuity of some holomorphic

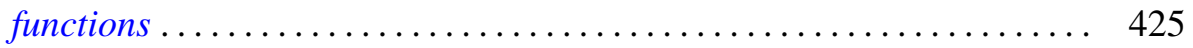

Frank Harary and Robert William Robinson, Generalized Ramsey theory.

IX. Isomorphic factorizations. IV. Isomorphic Ramsey numbers .......

Frank Harary and Allen John Carl Schwenk, The spectral approach to determining the number of walks in a graph...........

David Kent Harrison, Double coset and orbit spaces ..... . .

Shiro Ishikawa, Common fixed points and iteration of commuting

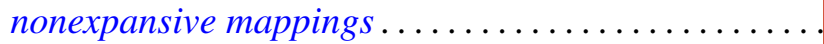

Philip G. Laird, On characterizations of exponential polynomials ........ 503

Y. C. Lee, A Witt's theorem for unimodular lattices ...........

Teck Cheong Lim, On common fixed point sets of commutative

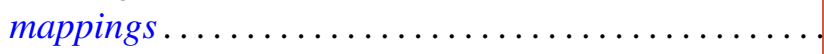

R. S. Pathak, On the Meijer transform of generalized functions ...

T. S. Ravisankar and U. S. Shukla, Structure of $\Gamma$-rings . . .

Olaf von Grudzinski, Examples of solvable and nonsolvable convolution

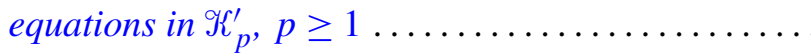

